\title{
The status of the second station of Taiwan Astroparticle Radiowave Observatory for Geo- synchrotron Emissions (TAROGE-II)
}

\section{T.C. Liu ${ }^{1,2}$}

LeCosPA center, National Taiwan University

R806, National Taiwan University \#.1. Roosevelt Rd., Taipei, Taiwan, 10617, ROC (Taiwan)

E-mail: TCLiuentu.edu.tw

(TAROGE Collaboration) C.-H. Chen ${ }^{1,2}$, C.-W. Chen ${ }^{2}$, Pisin Chen ${ }^{1,2}$, Y.-C. Chen' ${ }^{2}$, S.-Y. Hsu $^{2}$, J.-J. Huang ${ }^{2}$, M.-H. A. Huang ${ }^{1,3}$, Jiwoo Nam ${ }^{1,2}$, Jakub Ripa ${ }^{1}$, Y.-S. Shiao ${ }^{1,4}$, M.-Z. $\mathrm{Wang}^{2}$, \& S.-H. Wang ${ }^{1,2}$

${ }^{1}$ LeCosPA center, National Taiwan University, Taiwan(ROC)

${ }^{2}$ Physics Department, National Taiwan University, Taiwan(ROC)

${ }^{3}$ Department of energy engineering, National United University, Taiwan(ROC)

4. National Nano Device Laboratories, Taiwan(ROC)

Taiwan Astroparticle Radiowave Observatory (TAROGE) is an antenna array on the cliff of Taiwan's East coast to monitor ultra-high energy cosmic rays (UHECR, > $10^{19} \mathrm{eV}$ ). The antennas face the Pacific Ocean to detect radiowave signals emitted by the UHECR-induced air shower. The unique terrain of TAROGE site allows the antennas to collect both the directemission and the ocean-reflected radiowave signals induced by UHECR. So far two TAROGE stations have been deployed. The second TAROGE station (TAROGE-II), which consists of 6 dual-polarization log-periodic dipole antennas $(110-500 \mathrm{MHz})$, was installed on the $1100 \mathrm{~m}$ elevation near TAROGE-I in December of 2016. In this report, we introduce the basic concept of TAROGE, its data tacking, expected sensitivity, and future prospect. 


\section{Introduction}

The origin of ultra-high energy cosmic rays remains a fundamental and unsolved problem in astroparticle physics. Detecting the ultra-high energy cosmic rays (UHECRs) with energies above $\mathrm{EeV}$ is a challenge, it is limited by the low flux of UHECRs. Extending the detective area is the most intuitive solution of the cosmic rays experiments. For example, the detection area of the Pierre Auger Observatory (PAO) [1] covers 3,000 km², this size is the same order of magnitude as Luxembourg. The other giant ground array system, Telescope Array (TA) [2] occupies roughly the same area as New York City. All of the conventional surface detectors require a great land and the suitable climate for observation. To avoid the geometry constraints of experiment, the radio detection method became the promising techniques for UHECRs detection in last fifteen years. The main components of the radio emission that emitted by extensive air showers are geo-synchrotron radiation and radio Cherenkov. The electron-positron pairs of extensive air showers are deflected with opposite directions by earth's magnetic field and emit the coherent synchrotron emission named geo-synchrotron radiation. The other component is the Cerenkov radiation from the excess charges of the extensive air showers whereby the charges traveling faster than the speed of light in a dielectric medium [3] emit electromagnetic (EM) radiation. Askarayan postulated phenomenon in the 1960s so-called Askaryan effect [4,5]. The frequency range of the radio emission starts from $10 \mathrm{MHz}$ to a few hundred $\mathrm{MHz}$ [6-11]. The propagation distance of the electromagnetic wave within this frequency range can be up to several hundred kilometers and do not change with the weather condition. Hence the radio detector that is hundred kilometers far from the shower still can detect the radio pulse (geo-synchrotron radiation and Cherenkov radiation) form the extensive air shower. Therefore, one radio detector unit (antenna station) can cover huge detection area with high duty cycle. Several antenna-based experiments, such as ANITA [12], AREA [13], and LOPES [14] have demonstrated the capability of radio detection of UHECRs, even the potential detecting power of ultra-high energy cosmic neutrinos (UHECNs). For example, ANITA is a NASA Balloon-Borne observatory designed to investigate various phenomena related to UHECRs and UHECNs from $35 \mathrm{~km}$ high above the Antarctica. The effective detection area of ANITA is $\sim 10^{6} \mathrm{~km}^{2}$ with $700 \mathrm{~km}$ horizon distance. NASA LDB site already launched four flights at McMurdo, Antarctica, in the summers of 2006, 2008, 2014, and 2016, respectively. The ANITA observed a total of 20 UHECR events with a mean energy $\langle E\rangle=3 \times 10^{19} \mathrm{eV}$ and 2 of 20 UHECRs are the reflected events those reflected by the ice surface of Antarctica. The operation time of each flights is limited by the flight duration of the NASA Antarctic balloons and the typical duration is roughly $\sim 30$ days.

\section{The Taiwan Astroparticle Radiowave Observatory of Geo-synchrotron Emissions (TAROGE)}

By cloning the successful methodology of ANITAs, Taiwan Astroparticle Radiowave Observatory for Geo-synchrotron Emissions (TAROGE) places the antenna station at the top of mountains and face to the ocean to monitor both the direct and reflected signals from UHECRs (as Figure 1). The mountain's scale of the Taiwan's east coast is from a few hundred to two thousand meters order and extends about hundred kilometers. Although the altitude of the TAROGE is not as high as ANITAs, the detection area of TAROGE still can cover $1.3 * 10^{4} \mathrm{~km}^{2}$ 
with $160 \mathrm{~km}$ horizon radius and $60^{\circ} \mathrm{FOV}$ at $2 \mathrm{~km}$ altitude on the mountains. Comparing to ANITA, the most advantage of TAROGE is the operation time, it is almost $85 \%$ duty cycle of year-round operation; It restores the area loss of TAROGE.

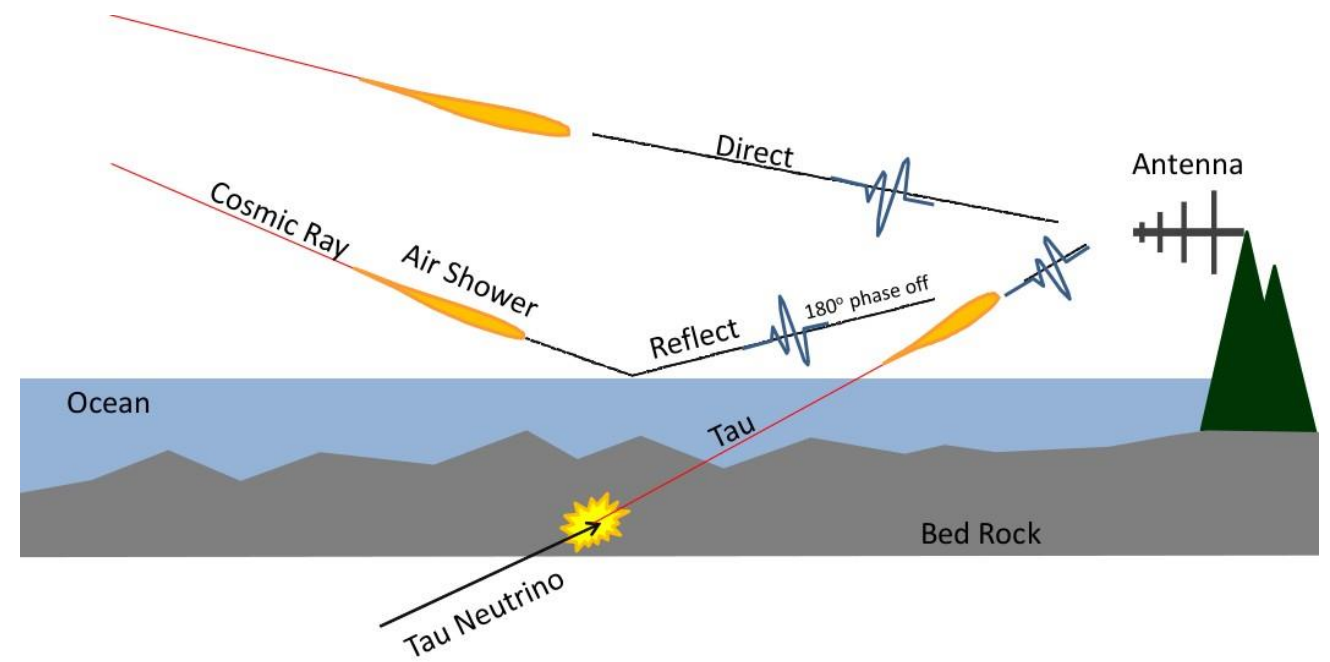

Figure 1: Detect strategy of the TAROGE. The antennas of TAROGE are placed on the thousand meters high cliff toward to the ocean to detect the radio emission from UHECRs and UHECNs.

The frequency band of the TAROGE antenna is from $110 \mathrm{MHz}$ to $300 \mathrm{MHz}$. Within this frequency range, sea water is a good conductors of electricity; the reflection coefficient of sea water of horizontally polarized radio wave and vertically polarized radio wave are $\sim 100 \%$ and $\sim 60 \%$, respectively. In comparison with the speed of radio wave, the instantaneous fluctuation of the ocean wave is negligible. Taiwan is a densely populated region, the population density of east coastal region of Taiwan is much lower than the western side. Figure 2 shows the power spectrum of the deep mountain area of Taiwan and the Figure 3 shows the continuous waveform (CW) noise peaks distribution of the TAROGE site. Although several noise peaks show up in the frequency band of TAROGE, but the rest frequency bands are the quite region (comparable to thermal- and Galactic-noises). Applying the RF filters and the multi-band trigger technique to TAROGE system, the CW noise cannot saturate our trigger system for long period. TAROGE became a more feasible experiment to choose the place.

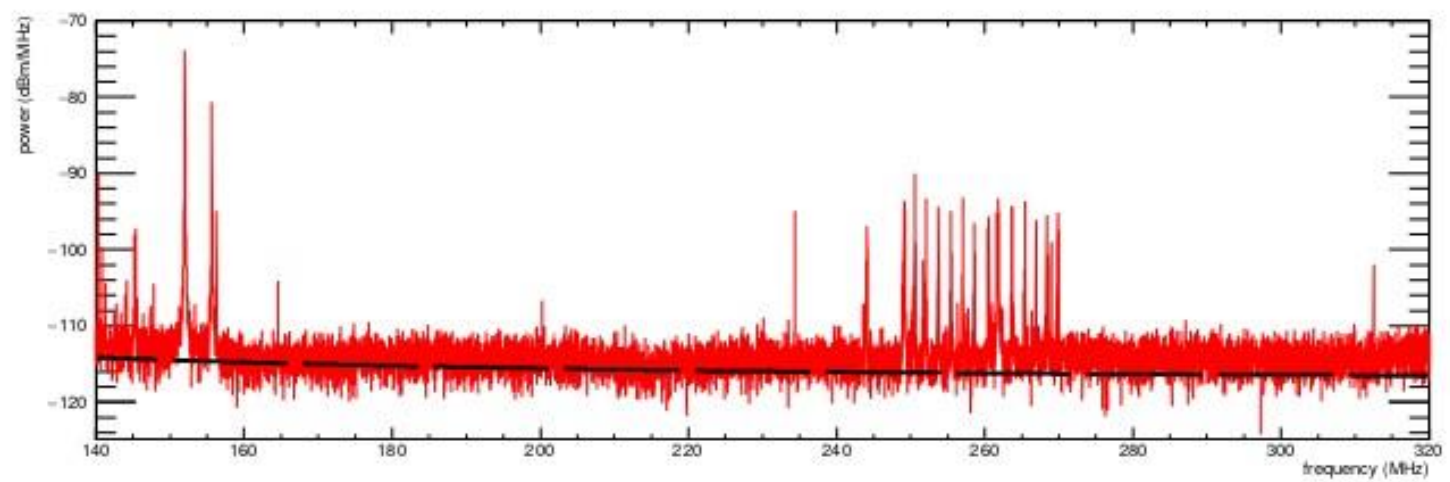

Figure 2. RF noise of the deep mountain area. We surveyed several sites of the eastern Taiwan, most of fixed noise peaks are identified. 


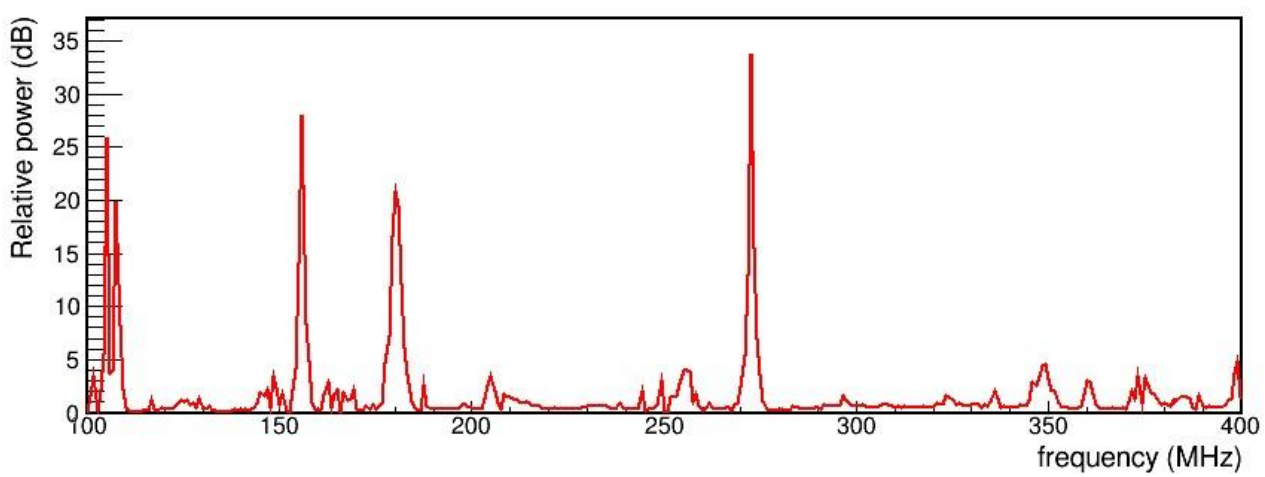

Figure 3. The noise peaks distribution in the TAROGE site. This distribution shows the frequency and the power of the CW noise those received by the TAROGE station. In our design, we have to notch the peaks to avoid the noise saturating our trigger system.

\subsection{The Status of TAROGE-I \& II Stations}

TAROGE-I is a prototype station which was aimed for systematic studies of RF enviroment in Taiwan. It established in the Yongshih mountain with $1,000 \mathrm{~m}$ elevation on July 2014 and starts its operation from the August 2014. The duty cycle is almost 100\% during winter and spring (during the typhoon season, the duty cycle drop to 65\%, due to the power loss and structure damage)[12]. TAROGE-I already retired after the systemic noise study and partially damaged by the typhoon in 2015. The upgrade system, TAROGE-II (Figure 4), was built at 1,150 m elevation at the same Mt. but 1,000 m apart from the TAROGE-I. Several items are upgrade for TAROGE-II as the following list

- The gain of the TAROGE-II antenna is $2 \mathrm{~dB}$ higher than the TAROGE antenna system;

- Merging two single log-periodic antennas (LPDA) to a dual-polarization LPDA, the new mechanic structure improves the antenna against the strong wind of typhoon and all antennas fully survive during the typhoon season 2016.

- The readout speed of DAQ increasing up to $40 \mathrm{~Hz}$.

- New design of the trigger board to avoid the pollution from the temporal appearance of $\mathrm{CW}$ noises.

- The independent photovoltaic (PV) power system for TAROGE. The radio detection technique requires the quite background to enlarge the dynamic detecting range. We design a solar power system for the next generation of TAROGE and test in the TAROGE-II. After the final setup of photovoltanic panels in winter of 2017, The TAROGE-II system can fully operate during a month without any power low sutdown issue. (First built in Nov 2015, temporal shutdown during 2016 due to site accessibility)

All upgades of TAROGE-II have been already completed and it started taking data with full operation since May 2017. In this study. we only show the preliminary analysis of TAROGE-II data of early May 2017 (10 days). The duty cycle of this period is about 85\% and the data implies that thunderstorms will generate some signals and recored by our system (Figure 5). The preliminary analysis bliends the data that coming angle below the horizon (reflected CR or 
tau neutrino events). Two candidates were discovered with the preliminary analysis (Figure 6) and the event rate is about one per five days.

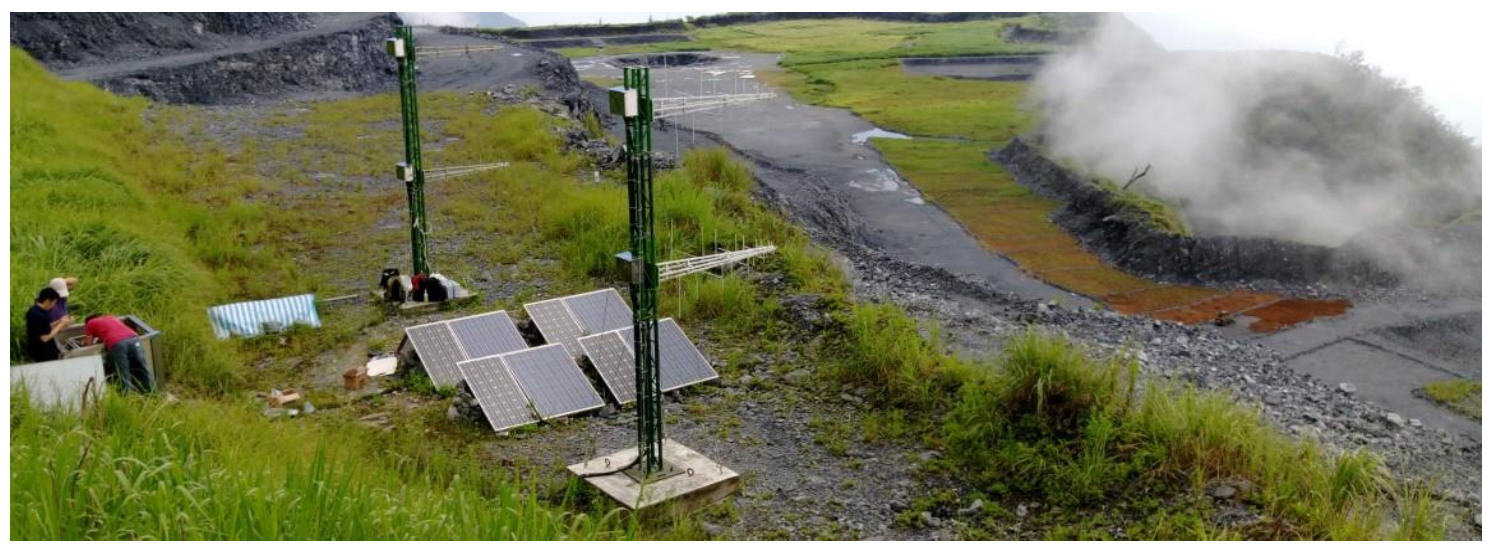

Figure 4. TAROGE-II tower structure. TAROGE-II includes three towers structure and each tower carry two dual-polarized LPDA antennas with a 2.5 meters spacing. This figure shows two towers and a 800W PV array system are placed in between of them.

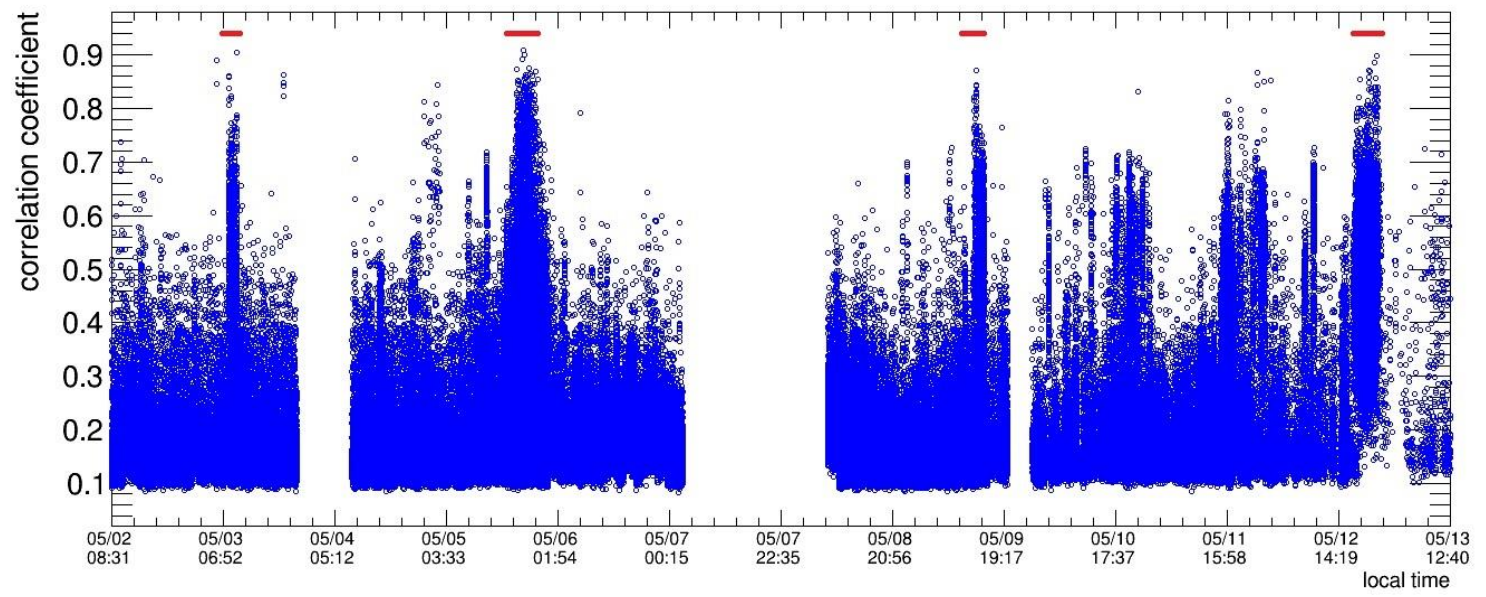

Figure 5. The signals are related to thunderstorm. During the summer, thunderstorm occurs in the Yongshih Mountain area frequently. The red bars denote the period during the thunderstorms, The TAROGE's event rate is highly correlative to the thunderstorm period.

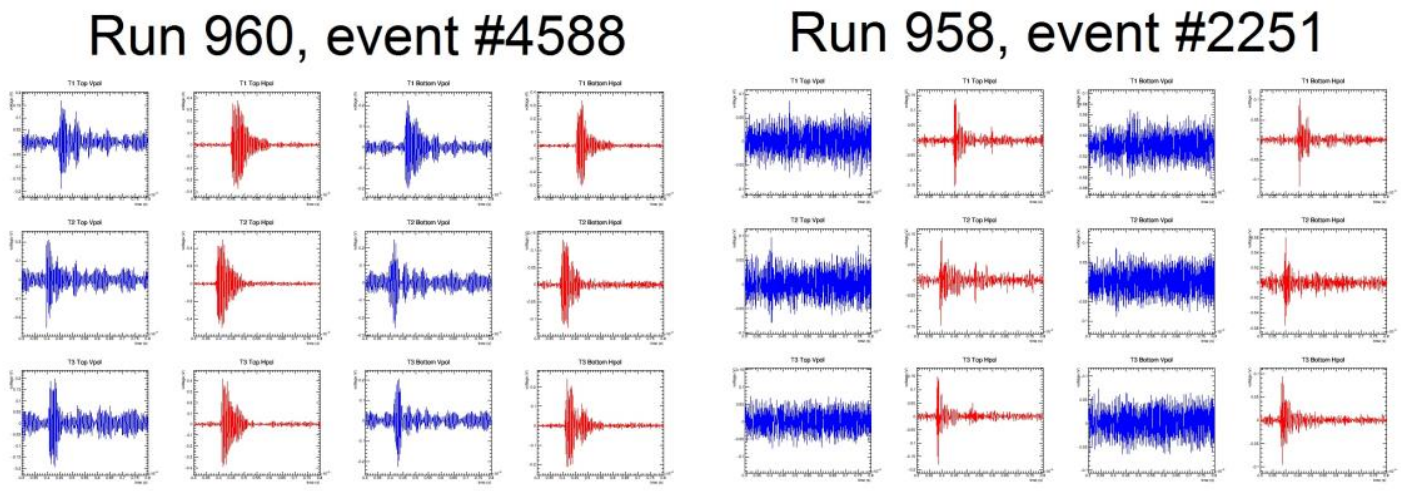

Figure 6. Two cosmic ray candidates. TAROGE-II starts its full time operation and taking data from May 2017. This figure shows the filtered waveform of the two candidates which are 
detected in early May. The blue and red lines denote the EM pulses, those detected by V-pol and H-pol antennas, respectively.

\section{Discussion}

TAROGE-I and TAROGE-II station were already built successfully during 2012-2016. The power system of TAROGE-II was upgraded in the spring season of 2017 and the duty cycle of TAROGE-II increasing up to $>85 \%$ from May 2017. The event rate during May 2017 is about $1 /(5$ days $)$, It is the same order of magitude as the initial simulation study. The simulation expected an event rate to be about 45 events/(year-station) at the $1,000 \mathrm{~m}$ altitude with assumptions of the $100 \%$ duty. The extension of TAROGE was funded by the Vanguard program of Ministry of Science and Technology (MOST), Taiwan, to build total seven stations in five years. Two new stations (TAROGE-III \& TAROGE-IV) is buliding from the summer 2017 and will complete in spring 2018 (Figure 7).

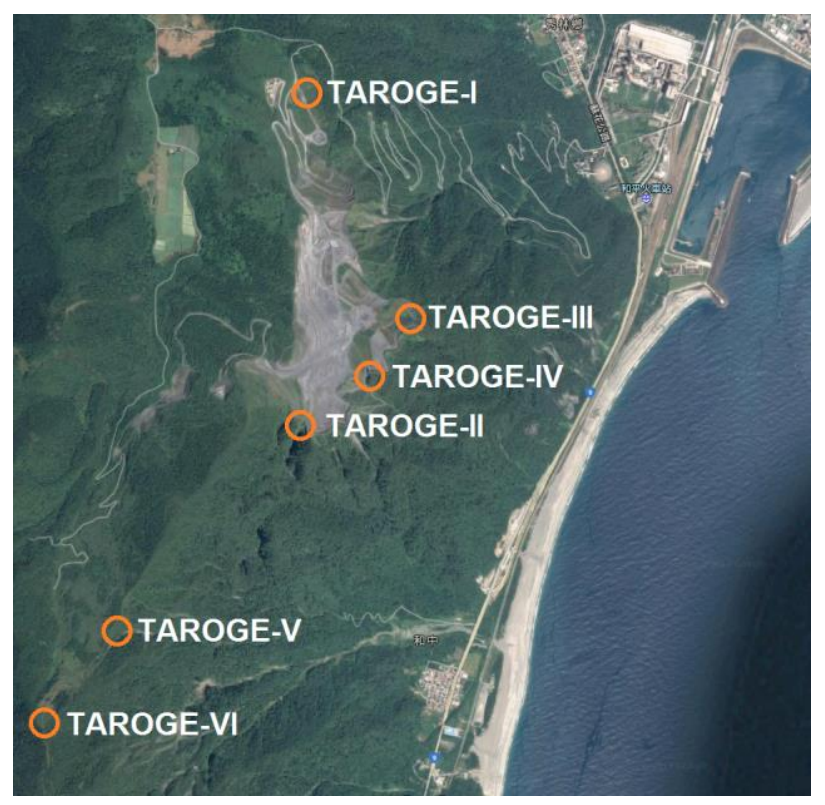

Retired station:

*2014: TAROGE-I (1000m)

Active station:

*2015: TAROGE-II (1050m)

In progressing:

*2017:TAROGE-III \& IV (1000m)

Next stations:

*2018 TAROGE-V \& VI (1350m)

Figure 7. The list of the TAROGE stations.

\section{Acknowledgments}

This work has been supported by the pioneer program of MOST, Taiwan. We gratefully thank Taiwan Cement Corporation and HePing elementary school for their invaluable support.

\section{References}

[1] Pierre Auger Collab., Nucl. Instr. Meth. A 798 (2010) 172.

[2] H.Kawai, et al. Nuclear Physics B: Proceeding supplement, 175-176 (2008), p221

[3] Falcke, H. \& Gorham, P. W. 2003, Astropart. Physics, 19, 477

[4] Askaryan G A. J. of Experimental and Theoretical Physics, 1965, 21: 658

[5] Askaryan G A. J. of Experimental and Theoretical Physics, 1962, 14: 441 
[6] Allan, H. R. 1971, Prog. in Element. part. and Cos. Ray Phys., Vol. 10, 171

[7] T. Huege and H. Falcke, Astropart. Phys. 24 (2005) 116.

[8] M. Ludwig and T. Huege, Astropart. Phys. 34 (2011) 483.

[9] O. Scholten, K. Werner, and f. Rusydi, Astropart. Phys. 29 (2008) 94.

[10] J. Alvarez-Muñiz, W.R. Carvalho, et al., Phys. Rev. D, 86, (2012) 123007.

[11] T. Huege, M. Ludwig, and C.W. James, AIP Conf.Proc. 1535 (2013) 128.

[12] ANITA Collab. (S. Hoover et al.), Phys. Rev. Lett. 105 (2010) 151101.

[13] Pierre Auger Collab. (T. Huege et al.), Nucl. Instr. Meth. A 617 (2010) 484.

[14] LOPES Collab. (H. Falcke et. al.). Nature, 435 (2005) 313.

[15] J. W. Nam et al, Int. J. Mod. Phys. D 25, 1645013 (2016) 